\title{
Factibilidad de la producción de pimienta (Piper nigrum) en La Virgen de Sarapiqui, Heredia, Costa Rica'
}

\author{
Feasibility of production of pepper (Piper nigrum) \\ in La Virgen de Sarapiquí, Heredia, Costa Rica
}

Dennis Monge-Cordero²

Tirsa Picado-Calvo 3

Fernando Morales-Abarca ${ }^{4}$

Fecha de recepción: 7 de marzo del 2013

Fecha de aprobación: I I de junio del 2013

Monge, D; Picado, T; Morales, F. Factibilidad de la producción de pimienta (Piper nigrum) en La Virgen de Sarapiqui, Heredia, Costa Rica.

Tecnología en Marcha.Vol. 26, № 4. Pág 72-84

I Trabajo elaborado como parte del curso de Preparación y Evaluación de Proyectos Agropecuarios, Universidad de Costa Rica.

2 Escuela de Economía Agrícola y Agronegocios, Universidad de Costa Rica, Costa Rica. Correo: dennis4II@gmail.com

3 Escuela de Economía Agrícola y Agronegocios, Universidad de Costa Rica, Costa Rica. Correo: tirpica@gmail.com

4 Escuela de Economía Agrícola y Agronegocios, Universidad de Costa Rica, Costa Rica. Correo: luis.morales@ucr.ac.cr 


\section{Palabras clave}

Rentabilidad; estudio técnico y financiero; TIR;VAN

\section{Resumen}

El objetivo de este trabajo es hacer un estudio de factibilidad para el cultivo de pimienta (Piper nigrum) en la Virgen de Sarapiquí, Costa Rica. Durante los meses de marzo a junio de 2012 se realizaron los estudios necesarios para determinar la viabilidad comercial, técnica y financiera del cultivo y producción de 2,5 hectáreas de pimienta en la Virgen de Sarapiquí, Heredia, Costa Rica. El estudio técnico mostró que la ubicación definida es adecuada para esta actividad. En cuanto a la viabilidad financiera, se concluye que el proyecto es factible ya que se obtuvo unVAN de $\not|5.53|$, I 27 y una TIR del 19,4\%.

\section{Key words}

Profitability; technical and financial study; IRR; NPV

\section{Abstract}

The aim of this study was to conduct a feasibility study for the pepper cultivation (Piper nigrum) in La Virgen de Sarapiquí, Costa Rica. During the months of March to June 2012, studies were conducted to determine commercial, technical and financial viability for the cultivation and production of 2.5 ha of pepper in La Virgen de Sarapiquí, Heredia, Costa Rica. The technical study shows that the selected location is suitable for this type of activity; as for financial viability, it is concluded that the project is feasible, this is determined by the resulting NPV of $\not|5,53|, \mid 27$ and IRR of $19,4 \%$.

\section{Introducción}

La producción de pimienta (Piper nigrum) en Costa Rica comenzó en los años 50 en la zona de La Tirimbina, en la Virgen de Sarapiquí, desde donde se expandió a otras áreas del país, especialmente las regiones Huetar Norte (Sarapiquí, San Carlos, Los Chiles, Upala y Guatuso) y atlántica (Talamanca, Siquirres, Pococi) (Consultorías Integradas Agropecuarias S. A., 2007) y en el Pacífico Sur (Golfito, Puerto Jiménez, San Isidro de Pérez Zeledón) (Aguilar y Bogantes, 199I).

Debido a que los productores no contaban con la asistencia técnica necesaria para enfrentar las enfermedades y plagas que atacaban el cultivo, el área de siembra de pimienta se redujo drásticamente, pasando de unas 500 hectáreas (ha) en los años 80 a unas 50 ha a inicios de los 90. En la actualidad, la mayor parte de la producción de pimienta se encuentra en los cantones de San Carlos, Sarapiquí, Guatuso y Upala, además de unas pocas plantaciones al sur del país, en Quepos (Consultorías Integradas Agropecuarias S. A., 2007).

La principal limitante para el desarrollo del presente trabajo es la falta de información relacionada con el producto, los pocos registros sobre volúmenes de producción, cantidades comercializadas y datos históricos de precios, con el fin de realizar un análisis más profundo de la situación del mercado.

El potencial y las ventajas que tiene la zona de Sarapiquí para el desarrollo de esta actividad la convierten en una oportunidad para la diversificación de la producción de los pequeños y medianos productores, de ahí la importancia de determinar la factibilidad técnica y financiera de la producción de pimienta en esta área.

\section{Materiales y métodos}

El estudio de factibilidad se realizó entre marzo y junio de 2012 en la comunidad de El Roble de la Virgen de Sarapiquí, en una finca que cuenta con 2,5 ha cultivadas de pimienta, propiedad de Emilia Carmona y Carlos Zamora. Se obtuvo información sobre el paquete tecnológico utilizado en la producción y se consultó bibliografía relevante sobre la actividad.

A través de La Compañía Agrícola Industrial PROPICA, S.A, la cual compra la pimienta en fresco a los propietarios de la finca estudiada, se obtuvo información relevante sobre datos históricos de precios y volúmenes de comercialización locales, mientras que para el mercado internacional se consultaron las bases de datos de la Organización 
de las Naciones Unidas para la Alimentación y la Agricultura (FAO).

En cuanto al estudio de mercado, por lo general, el concepto de estudio de mercado se identifica con la definición tanto del precio al que los consumidores están dispuestos a comprar como a la demanda, según indican Sapag y Sapag (2008). En esta investigación se estudió la comercialización del producto, los proveedores, precios actuales y futuros y proyecciones del mercado, entre otros aspectos.

El estudio técnico se basa en la búsqueda de la información necesaria para optimizar los recursos para la producción y cuantificar el monto de las inversiones y de los costos de operación, además, se determinan los requerimientos de equipo y prácticas a desarrollar para la producción de pimienta.

También se identifican aspectos legales, ambientales y administrativos que pueden afectar los niveles de flujos de caja del proyecto, para que sean incluidos y analizados en las diferentes herramientas del estudio financiero.

El estudio financiero es uno de los elementos más importantes dentro de un estudio de factibilidad, ya que analiza la viabilidad financiera del proyecto y la rentabilidad generada por la actividad.

De esta forma se utilizan los flujos de caja nominales aplicados a un horizonte temporal de 10 años, los cuales se evaluaron a través de los indicadores de Valor Actual Neto (VAN), Tasa Interna de Retorno (TIR) y Período de Recuperación de la Inversión (PRI).

Además, se realizó un análisis de sensibilidad unidimensional para determinar los precios mínimos aceptables para la estabilidad del proyecto, así como los costos máximos anuales en los que se podría incurrir sin que deje de ser rentable.

El análisis de sensibilidad bidimensional permitió determinar el efecto que pueden tener en los indicadores de la TIR y del VAN los aumentos y disminuciones de los niveles de producción, costos y precios.

\section{Resultados y discusión}

Los resultados del estudio se presentan en tres apartados: estudio de mercado, estudio técnico y estudio financiero y de análisis de sensibilidad.

\section{Estudio de mercado}

La planta de pimienta es una liana perenne y trepadora que puede alcanzar una altura mayor a 10 metros y necesita un tutor para su crecimiento. La liana está compuesta por internudos en los que se producen raíces adventicias capaces de asirse para permitir el ascenso. La pimienta presenta tres tipos de crecimiento: los estolones, que crecen a ras del suelo, el tallo vertical, y las ramas laterales, en las que se producen los frutos en racimo (EARTH, 20 I I).

La planta tiene tallos principales, de los cuales surgen las ramas fructíferas y los tallos al nivel del suelo, sus hojas son pecioladas, simples y alternas, sus inflorescencias son espigas y colgantes. Pueden tener de 3 a $15 \mathrm{~cm}$ de largo, albergando de 50 a 150 flores diminutas, de color verde amarillento y dispuestas en espiral a lo largo del raquis, en donde luego se formarán los frutos, drupas que al madurar pasan de color verde a amarillo y luego a rojo (EARTH, 20 I I).

Para la pimienta existen varios procesos de beneficiado. En este caso, el estudio se centra en la producción de pimienta fresca para su industrialización con el fin de obtener pimienta negra.

En las zonas productoras en Costa Rica, la variedad más utilizada es la Balankotta, que se caracteriza por una producción aproximada de hasta $4 \mathrm{~kg}$ secos por año. La técnica de reproducción es asexual, mediante la utilización de acodos, con el fin de utilizar los mejores materiales genéticos (EARTH, 20ll).

La pimienta fresca que se intenta producir en este proyecto se venderá a la empresa PROPICA y su transporte se realizará en sacos de $20 \mathrm{~kg}$ y $40 \mathrm{~kg}$ hasta la planta; por lo tanto es un producto intermedio que funciona como materia prima para el proceso de industrialización.

Según datos de la FAO, en 2009, en el mercado internacional de la pimienta, el país que produjo mayor cantidad fue Vietnam, seguido por Indonesia e India. Estos países, junto con Brasil, son los principales exportadores. Por otra parte, los países que más importan son Estados Unidos, Alemania e India, este último es uno de los mayores comerciantes de especias en el mundo, por lo que aparece entre los mayores exportadores e importadores.

\section{Mercado nacional}

En Costa Rica, la Región Huetar Norte es la que presenta la mayor cantidad de producción de 
pimienta, y se caracteriza por ser pequeñas explotaciones, de alrededor de I ha. El cantón con mayor área sembrada para el año 2007 fue Sarapiquí, con más de 100 ha, ubicadas en su mayoría en la comunidad de Rancho Chilamate.

En la figura I se presenta la cantidad de toneladas de pimienta producidas en el país en el período 1989-2009, el cual se caracterizó por constantes variaciones, lo que dificulta la determinación de pronósticos.

La figura 2 muestra el comportamiento del volumen de las exportaciones e importaciones de pimienta en Costa Rica desde 1994 hasta 2009. Se observa

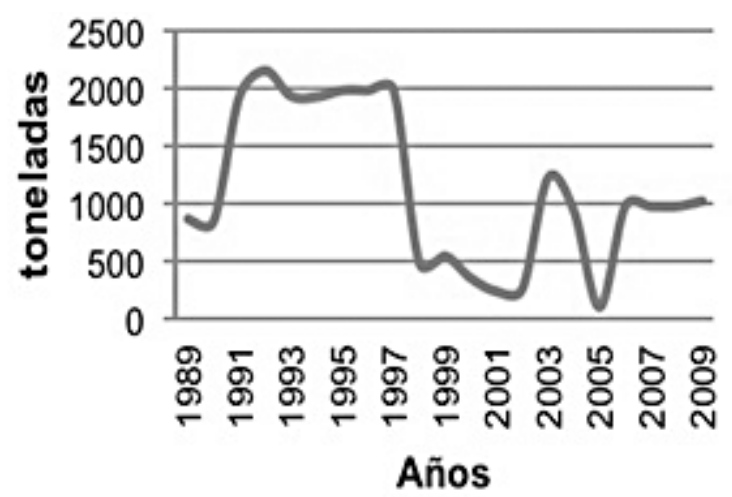

Figura I. Producción de pimienta, en toneladas para el período 1989-2009. Costa Rica. FAO, 2012.

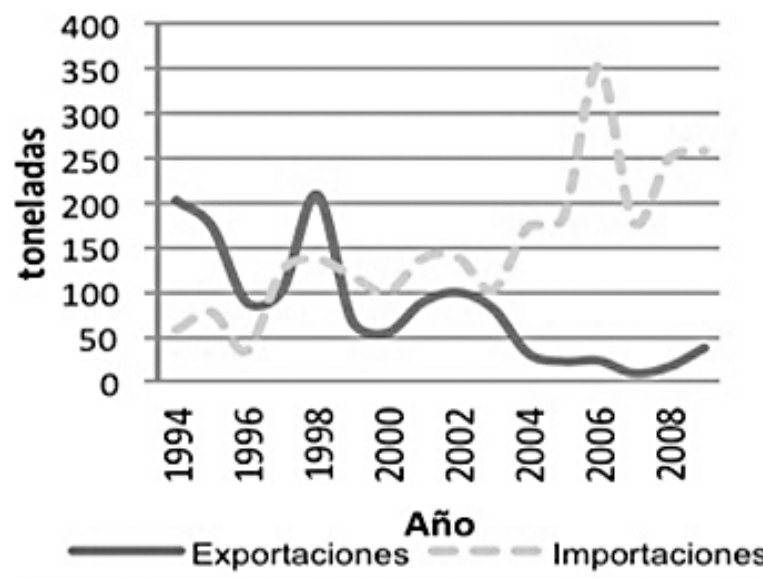

Figura 2. Volumen de las exportaciones e importaciones de pimienta, en toneladas, en el período 1994-2009. FAO, 2012. un aumento en las importaciones y una disminución en las exportaciones.

La demanda está determinada por el precio del producto, la calidad y el contenido de piperina, que es el principio activo de la pimienta. Costa Rica tiene una ventaja competitiva con relación a los demás oferentes, pues sus niveles de piperina superan el 7\%, mientras que los demás países apenas alcanzan el 5\%. Esta característica es muy apreciada por la industria tanto nacional como internacional (Consultorías Integradas Agropecuarias S.A., 2007).

Con respecto a la demanda doméstica de la empresa PROPICA, el Ingeniero Luis Fernando Mora indica que para el período 2004-20 I I se presentaron variaciones debido principalmente al efecto de los precios internacionales. La cantidad recibida por esta empresa en este período se ubicó entre las 170 y 380 toneladas anuales.

La demanda aparente estará conformada por la cantidad de pimienta que se produce en el territorio nacional, sumada a las importaciones y excluyendo las exportaciones, mediante la siguiente fórmula:

$$
D A=P N+M-X
$$

En donde DA corresponde a demanda aparente, $\mathrm{PN}$ es la producción nacional y $\mathrm{X}$ y $\mathrm{M}$ son las exportaciones e importaciones respectivamente. En la figura 3 se observa un comportamiento fluctuante de la demanda aparente de pimienta durante el período 1989-2009.

En la zona de Sarapiquí, la pimienta alcanza al tercer año de la siembra una producción de 2,6 t/ha, para

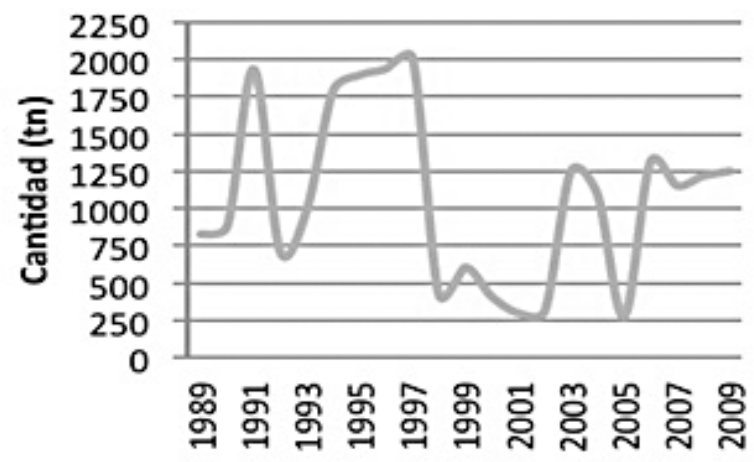

Años

Figura 3. Demanda de pimienta, en toneladas, en el período 1989-2009. Costa Rica. FAO, 2012. 
el cuarto año 5,2 t/ha, el quinto año 10,3 t/ha y se estabiliza a partir del sexto año en aproximadamente I2,9 t/ha (EARTH, 20 I I).

Al igual que en el caso de la demanda, en la figura 4 se observan las fluctuaciones en la oferta de pimienta, causadas por la inestabilidad de la producción.

Los precios del mercado nacional pagados al productor se calcularon mediante la división del valor total anual de la producción de pimienta entre la cantidad de producción, ambos registrados por la $\mathrm{FAO}$, con el fin de obtener un indicativo de los precios promedio del kilogramo de producto.

En el período de 1989 a 2009, el precio promedio pagado al productor de pimienta fue de $\not|062,7|$ por kilogramo, alcanzando un precio máximo de $\not 1064,80$ en 2005, que como se indicó anteriormente fue el año con menor producción. Según este análisis, las variaciones en el precio son pequeñas y se han mantenido muy constantes a través del tiempo. Se debe entender que estos precios son el promedio del total de precios nacionales.

En el caso de este productor, al poseer un contrato fijo con PROPICA, es importante analizar los precios que la empresa paga por kilogramo de producto, los cuales van desde $\$ 0,65$ en el año 200 I hasta $\$ 1,65$ en junio de 2012 .

\section{Proyecciones del mercado}

Las proyecciones de las variables de oferta, demanda y precios se realizaron mediante la utilización del método de promedios móviles, con el fin de

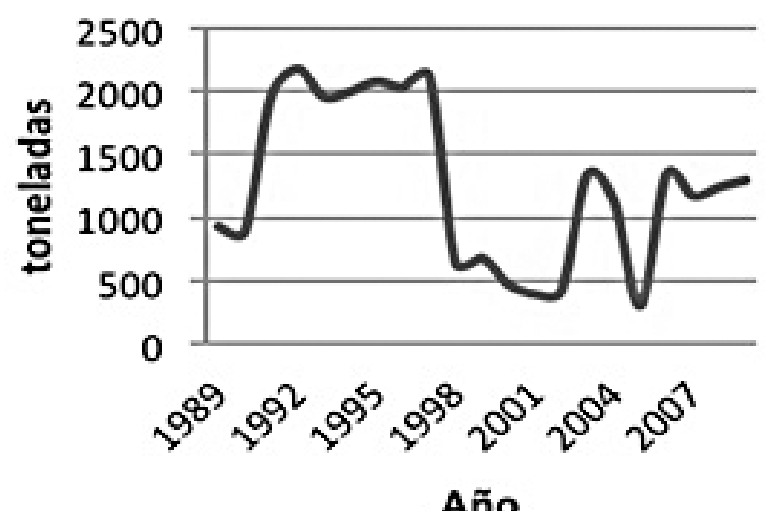

Figura 4. Oferta de pimienta, en toneladas, en el período 1989-2009. Costa Rica. FAO, 2012. hacer el análisis futuro de la oferta y la demanda de pimienta; dichos resultados se presentan en la figura 5 y el cuadro I. En la figura 5 se aprecian las proyecciones de la oferta y la demanda entre 2010 y 2022, donde se espera un comportamiento relativamente estable de dichas variables.

Para este proyecto, el único cliente es la empresa PROPICA, que a la vez es la principal proveedora de Griffith Spices y, según estimaciones, únicamente abastece el 30\% de la demanda de su comprador, por lo que existe una aparente demanda insatisfecha (Segura, 2007).

\section{Análisis de la comercialización}

En Sarapiquí, la comercialización de la pimienta se realiza de forma directa entre el productor y la empresa compradora, PROPICA S.A. Como se puede observar, la cadena de comercialización y los actores que participan en ella es muy sencilla, son solo dos, el productor o vendedor y PROPICA (Segura, 2007).

PROPICA proporciona un contrato de compra que le permite al productor tener un comprador estable por 15 años. El contrato estipula un precio mínimo de compra, lo que ofrece un margen de seguridad a los productores de que su producto será comercializado, especialmente a futuro. El embalaje se da en sacos de $20 \mathrm{~kg}$ y $40 \mathrm{~kg}$ de peso de cada uno.

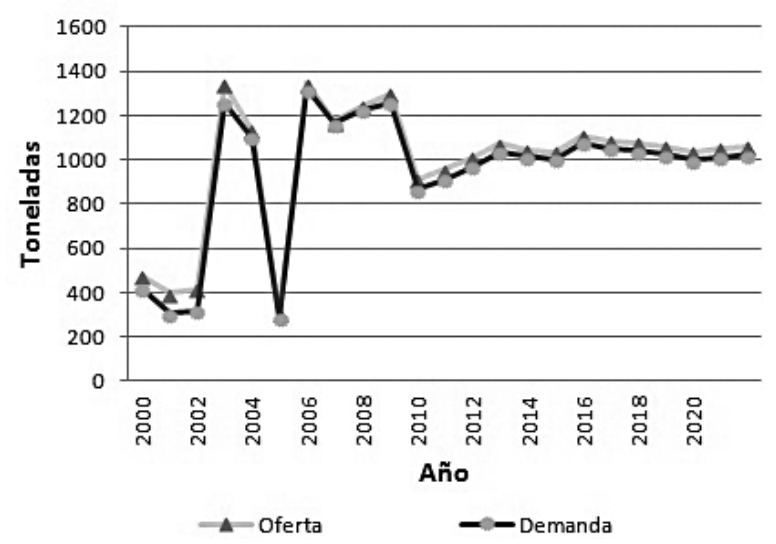

Figura 5. Comportamiento histórico y proyectado de la demanda y oferta de pimienta, en toneladas, en el período 2000-2022, Costa Rica. Elaboración propia con datos de FAO, 2012. 
Cuadro I. Proyecciones del precio (colones), oferta y demanda de pimienta en el período 2010-2022, Costa Rica. Elaboración propia con datos de $\mathrm{FAO}, 2012$.

\begin{tabular}{|c|c|c|c|}
\hline Año & $\begin{array}{c}\text { Precio }(\not l \\
\mathrm{kg})\end{array}$ & $\begin{array}{c}\text { Oferta } \\
\text { (toneladas) }\end{array}$ & $\begin{array}{c}\text { Demanda } \\
\text { (toneladas) }\end{array}$ \\
\hline 2010 & 1062,9 & 913,4 & 866,4 \\
\hline 2011 & 1063,0 & 957,4 & 911,2 \\
\hline 2012 & 1063,0 & 1013,6 & 971,7 \\
\hline 2013 & 1063,0 & 1072,9 & 1036,8 \\
\hline 2014 & 1063,0 & 1045,9 & 1014,4 \\
\hline 2015 & 1063,0 & 1037,2 & 1005,8 \\
\hline 2016 & 1062,8 & 1110,3 & 1077,9 \\
\hline 2017 & 1062,9 & 1086,6 & 1053,4 \\
\hline 2018 & 1062,9 & 1077,9 & 1042,5 \\
\hline 2019 & 1062,9 & 1061,3 & 1024,0 \\
\hline 2020 & 1062,9 & 1037,7 & 1000,4 \\
\hline 2021 & 1062,9 & 1050,1 & 1013,8 \\
\hline 2022 & 1062,9 & 1059,4 & 1024,1 \\
\hline
\end{tabular}

El productor puede transportar directamente su pimienta a la empresa compradora o bien se lleva toda a un centro de acopio y un transportista privado traslada el producto. Los volúmenes de entrega varían desde $30 \mathrm{~kg}$ hasta $1000 \mathrm{~kg}$ por productor (Segura, 2007).

\section{Políticas de ventas y de precios}

Los precios están determinados por el efecto de la oferta y la demanda internacional, donde el importador es el que influye más en el comportamiento a través de las respectivas negociaciones. En este caso, la empresa PROPICA asegura un precio mínimo mediante el contrato que hace con el productor (Segura, 2007).

\section{Posibilidades del proyecto}

Mediante un análisis FODA se determinaron las posibilidades del proyecto según las fortalezas, oportunidades, debilidades y amenazas.
Entre sus mayores fortalezas está la experiencia en la producción, la estabilidad del mercado y los términos de negociación, así como las buenas condiciones de la zona para el cultivo. Como oportunidades se tiene principalmente la buena calidad de la pimienta de Sarapiquí y la existencia de una demanda insatisfecha.

Por otra parte, entre las debilidades más importantes están la falta de capacitación técnica, la deficiencia en el manejo de la finca y la inexistencia de registros de producción y manejo. Además, el proyecto puede verse amenazado por factores externos como la inestabilidad en la producción, la dependencia de un solo comprador, los efectos del cambio climático y la poca variabilidad genética del cultivo.

\section{Estudio técnico}

\section{Tamaño y localización del proyecto}

Es necesario determinar el tamaño y la ubicación del proyecto, ya que estos factores influyen en el cálculo de las inversiones y los costos de operación; además se deben tomar en cuenta otros factores como la demanda y la disponibilidad de los insumos.

\section{Topografía de suelos}

Para su siembra, la pimienta prefiere suelos de origen aluvial, sueltos y con buen drenaje. Se recomienda un $\mathrm{pH}$ de 5,5, a 6,5 con buen contenido de materia orgánica, con pendientes de 0\% a 30\% (EARTH, 20I I), características que cumple la topografía de suelos predominante en la zona de El Roble de Sarapiquí, por lo que es un factor positivo para el desarrollo de la actividad en el lugar.

\section{Nutrición}

\section{Requerimientos nutricionales}

Al momento de la siembra, en el fondo del surco se aplican dos sacos de fertilizante de la fórmula 10-3010 por hectárea. A los seis meses, el cultivo requiere de dos aplicaciones de la fórmula |8-5-|5-6-1.2, equivalente a 1,5 sacos por ha; al año requiere de tres aplicaciones de |8-5-| 5-6-I.2 (seis sacos) y una aplicación de I saco de úrea; al tercer año requiere de dos aplicaciones de seis sacos de 18-5-15, un saco de urea y tres sacos de |5-3-3|. A partir del cuarto año, requiere una aplicación de tres sacos de | 8-5- 15 y luego tres aplicaciones más de cuatro sacos por ha (Bertsch y Méndez, 20I I). 


\section{Sanidad}

\section{Combate de malezas}

El combate de malezas debe hacerse mediante chapeas, previa rodajea a mano, para no dañar las raíces, que son muy superficiales y susceptibles al ataque de fitoptora o fusarium. Estas chapeas deben realizarse entre los seis meses o el año de edad de la plantación; se puede aplicar herbicida quemante en el área de la rodaja. La rodaja se debe deshierbar manualmente para no dañar las raíces y evitar las enfermedades (Aguilar y Bogantes, 199|).

Poda

Cuando la planta de pimienta alcanza entre 40 y 60 $\mathrm{cm}$ de altura y tiene ocho a diez nudos, aproximadamente a los seis meses de establecida en el campo, se le debe realizar una poda inicial para inducir la producción de brotes laterales, la cual consiste en cortar la parte superior de la liana y dejar solo seis nudos (Aguilar y Bogantes, 199|).

Una vez que ocurre el rebrote, se seleccionan los cuatro o seis mejores tallos, los cuales se deben amarrar paralelos al tutor, sin permitir que se crucen, con un material de rápida degradación. Esta práctica es necesaria en la pimienta para darle formación a la planta, eliminar los tallos más débiles y permitir el crecimiento de los más fuertes (Aguilar y Bogantes, |99|).

\section{Rendimientos}

El grano destinado a pimienta negra produce 33\% de peso seco, con respecto al peso de la pimienta fresca. Para pimienta blanca, el rendimiento es de $30 \%$ con respecto a la pimienta fresca (Aguilar y Bogantes, |99|).

En Costa Rica, el rendimiento oscila entre 1600 a

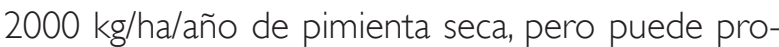
ducir hasta $4.000 \mathrm{~kg} / \mathrm{ha} / \mathrm{año}$ cuando se le brinda el mantenimiento adecuado a la plantación y la planta produce durante seis a ocho meses al año (Aguilar y Bogantes, 1991).

Las cosechas más importantes se recogen entre el tercero y séptimo año de edad y la vida productiva de la planta, con una producción de I a $2 \mathrm{~kg}$, puede durar de 15 a 20 años, aunque es aconsejable renovar después de los 10 (Aguilar y Bogantes, 1991).

Aspectos económicos
La producción de pimienta en esta zona no requiere inversiones de alta tecnología, ya que la mayoría de las labores se realizan manualmente debido a la naturaleza y complejidad del cultivo.

Por lo tanto, la inversión en maquinaria y equipo para la producción se estima en $\$ 622000$ y está constituida por una motobomba, una motoguadaña y algunas otras herramientas menores, lo cual genera una depreciación anual de $\$ 62$ 200, con una vida útil de 10 años.

La obra física consiste en la construcción de una cerca perimetral que brinde protección al cultivo y cuyo costo aproximado es de $\not|l| 94265$, con una vida útil de 20 años, por lo que su depreciación anual es de $\not 597$ 7 3.

También se requiere construir una bodega para guardar insumos y equipo, además de almacenar ciertas cantidades de cosecha. Esta estructura tiene un costo de $\not 1368000$ y su depreciación anual es de $\not 27360$, con una vida útil de 50 años.

Por otra parte, los costos de mano de obra para el proyecto se estiman según el balance de horas por labor realizada, siendo el primer año el período en el que más cantidad de horas-hombre se requieren, con un total de 2076; en los demás años de evaluación este número varía entre 1200 y 1400 horas por año, con un valor unitario de $\not 985,48$. Además, se debe incurrir en gastos de insumos agrícolas y de transporte, los cuales van a variar según el estado de la producción.

\section{Estudio financiero y análisis de sensibilidad}

Los ingresos generados por la producción y venta de pimienta en este proyecto, según el sistema de comercialización del producto, se pueden dividir en dos rubros: la venta de la pimienta fresca y la venta de la pimienta seca. En el primer caso, la pimienta que se comercializa fresca se vende a PROPICA, como se detalló en el estudio de mercado, a un precio actual de $\$ 1,65$ por kilogramo, mientras que la pimienta que ha sobrepasado el grado de madurez se pone a secar y posteriormente se vende a la misma empresa a un precio de $ф 2700$ el kilogramo, generando así un ingreso adicional.

En este proyecto los costos se determinan según los requerimientos de mano de obra, materiales y otros gastos, que se estiman según lo indicado en 
el estudio técnico. Además, se agregan los costos necesarios para la mitigación de los impactos de la producción sobre el medio ambiente.

La depreciación de los activos también debe incluirse para el cálculo del flujo de caja, ya que se debe restar a la utilidad bruta, para luego determinar el monto del impuesto sobre la renta que se debe pagar, sin embargo, al no corresponder a un gasto desembolsable real de dinero, se deben sumar para obtener el flujo de caja con que va a contar el proyecto.

El impuesto sobre la renta utilizado para este proyecto se estimó mediante la aplicación de las tasas que determina la Dirección General de Tributación Directa del Ministerio de Hacienda para las personas físicas con actividad lucrativa.

El método utilizado para el cálculo de la inversión en capital de trabajo es el del período de desfase, el cual comprende la suma de los costos operativos de los primeros tres años de la producción, que en total es de $\not 24236812$.

Mediante el método económico (Sapag y Sapag, 2008), se determina que el valor de desecho del presente proyecto al final del período de evaluación es de $\not 96489549$.

Para establecer la tasa de descuento se utiliza como referencia el valor de la tasa básica pasiva del Banco Central de Costa Rica, que es de $9,75 \%$ en el primer semestre de 2012. A esta tasa se le agrega una prima por riesgo de la actividad agrícola, la que se estimó en un $5 \%$, por lo que la tasa de descuento a utilizar para la evaluación es del 14,75\%.

La tasa por riesgo es relativamente alta, debido a que la actividad agrícola está sujeta a una serie de riesgos relacionados directamente con la naturaleza de la producción, como son los efectos climatológicos, de plagas y enfermedades y precios, entre otros.

La estabilidad de los flujos a partir del sexto año coincide con el alcance máximo de la producción estimada.

Para este análisis se utilizaron los indicadores del Valor Actual Neto (VAN), la Tasa Interna de Retorno (TIR) y el Período de Recuperación de la Inversión (PRI). En el cuadro 2 se presenta un desglose de los costos variables del proyecto de producción de pimienta, para un período de 10 años.

Este proyecto genera un VAN de $\not 1553 \mid 127$ y una TIR del 19,4\%, que al compararla con una tasa de descuento del 14,75\% evidencia que el proyecto es rentable. Tanto la TIR como el VAN son positivos.

Como un indicador adicional se muestra el PRI, el cual consiste en el tiempo que se tarda en que los flujos de efectivo recuperen el dinero invertido. El proyecto presenta un PRI de siete años, tomando en cuenta que los ingresos se empiezan a percibir a partir del tercer año.

\section{Análisis de sensibilidad}

Uno de los aspectos en los que el proyecto se puede ver afectado es el precio y sus variaciones, por lo tanto determinar el precio mínimo aceptable es necesario e importante. El precio que PROPICA pagaba al productor en 2012 era de $\$ 1,65$ por kilogramo de producto fresco, lo que equivale a $\Varangle 831,45$, según el tipo de cambio del Banco Central de Costa Rica al 19 de junio de 2012 ( $\$ 503,93$ por dólar). En este caso, el precio mínimo aceptable para el proyecto es de $₫ 705,08$ por kilogramo.

Con base en el precio pagado por PROPICA al productor, y en caso de ocurrir cambios en los costos de producción, los costos máximos anuales aceptables se pueden observar en el cuadro 3.

Con base en un análisis bidimensional, se evaluaron los escenarios para el proyecto en los cuales los precios, los costos y la producción se aumentan o disminuyen en $20 \%$ y $40 \%$.

Con un nivel de producción de $6.500 \mathrm{~kg} / \mathrm{ha} / \mathrm{año}$, la TIR se vería afectada hacia la disminución si los costos de producción aumentan 40\%, no obstante, ese cambio no se considera sensible porque la variación en la TIR es menor a la variación en los costos. Por otra parte, si el nivel de costos permanece constante y el precio disminuye 20\%, la TIR sería del $12,77 \%$, lo cual es menor a la tasa de descuento del proyecto.

Si el precio por kilogramo de pimienta disminuye $20 \%$, entonces la TIR disminuiría en un $36 \%$, por lo tanto, al ser el cambio en la tasa de retorno mayor al cambio en el precio, se considera que la TIR es sensible ante cambios en el precio. 
$\frac{0}{2 ㅇ ㅡ ㄴ ~}$

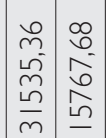

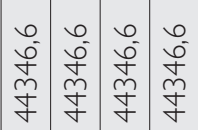

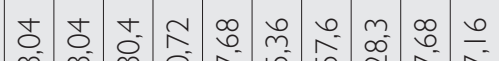

选

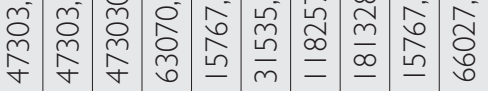

$a$
0
$i c$
$<$

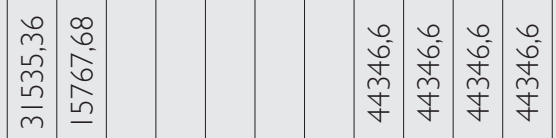

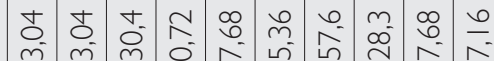

m

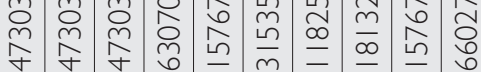

$\infty$
는
$<$

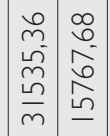

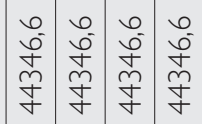

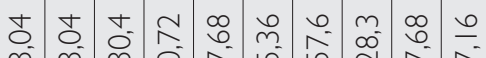

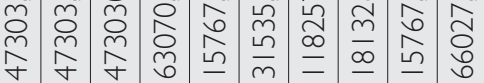

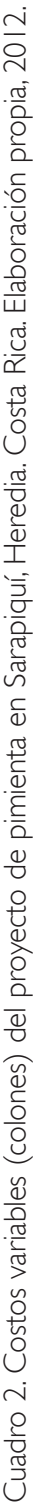

1은

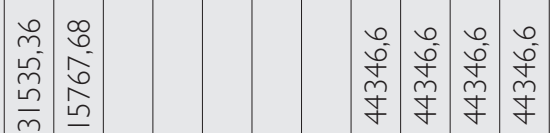

t

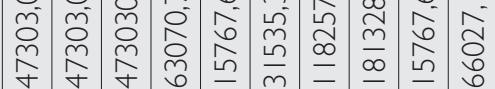

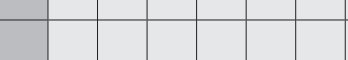

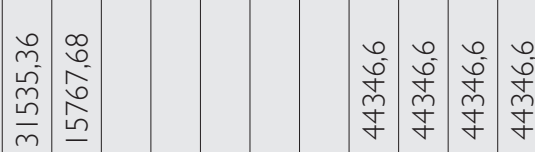

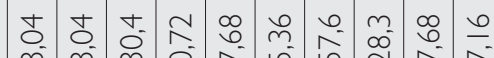

0
0
2
2
2

는 느

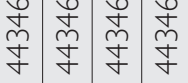

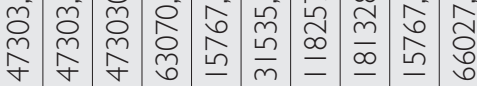

in

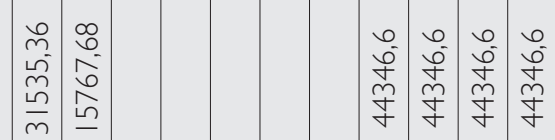

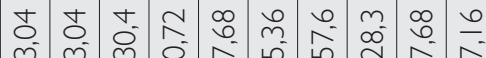

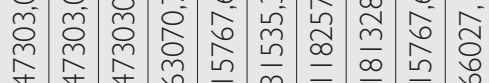

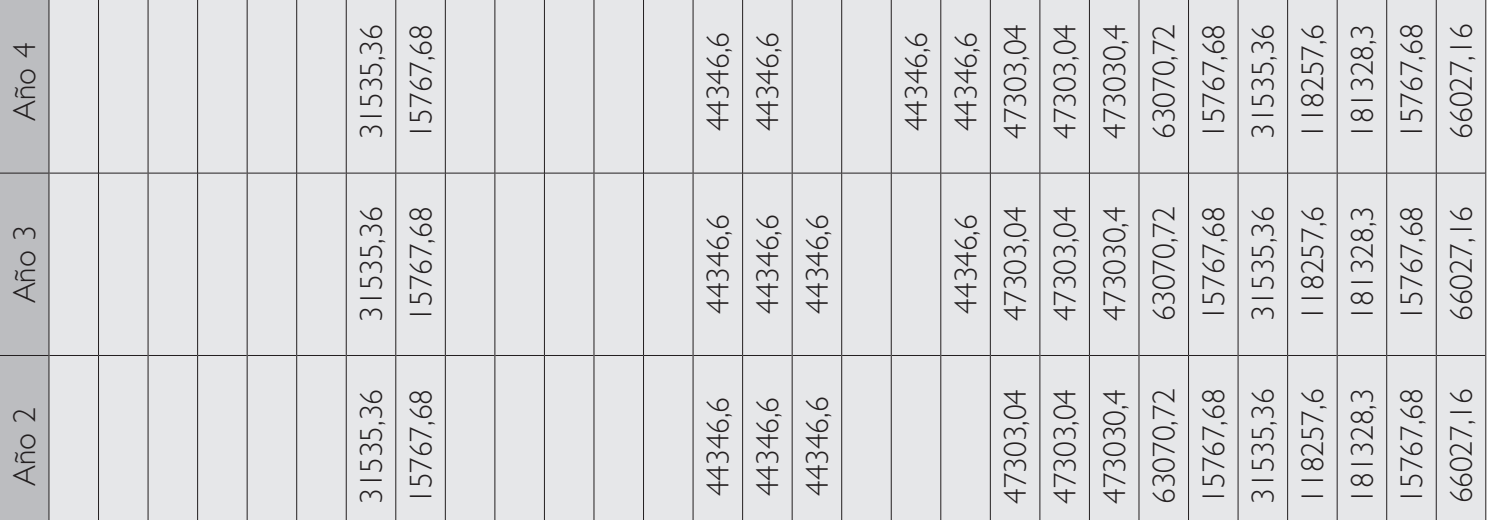

ఫ m

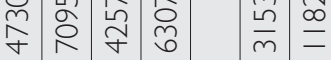

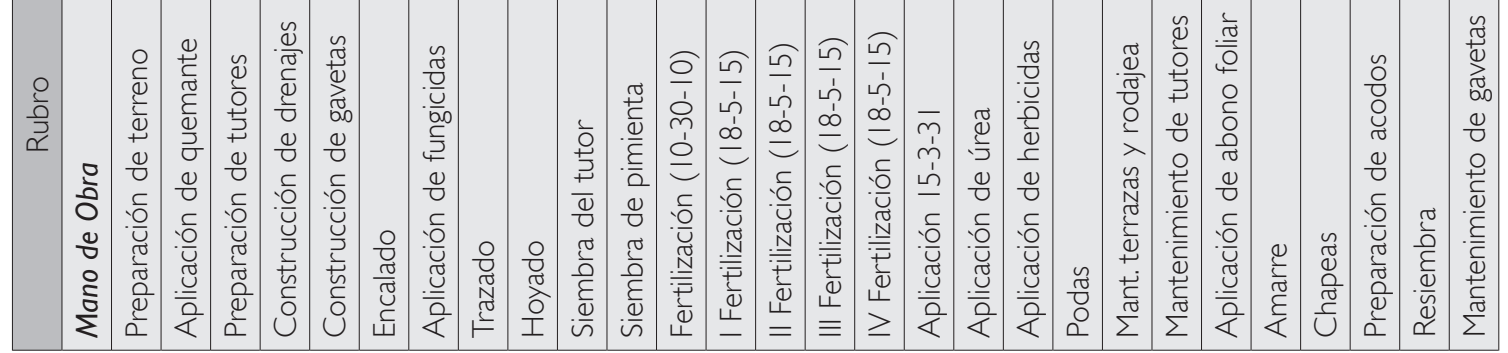




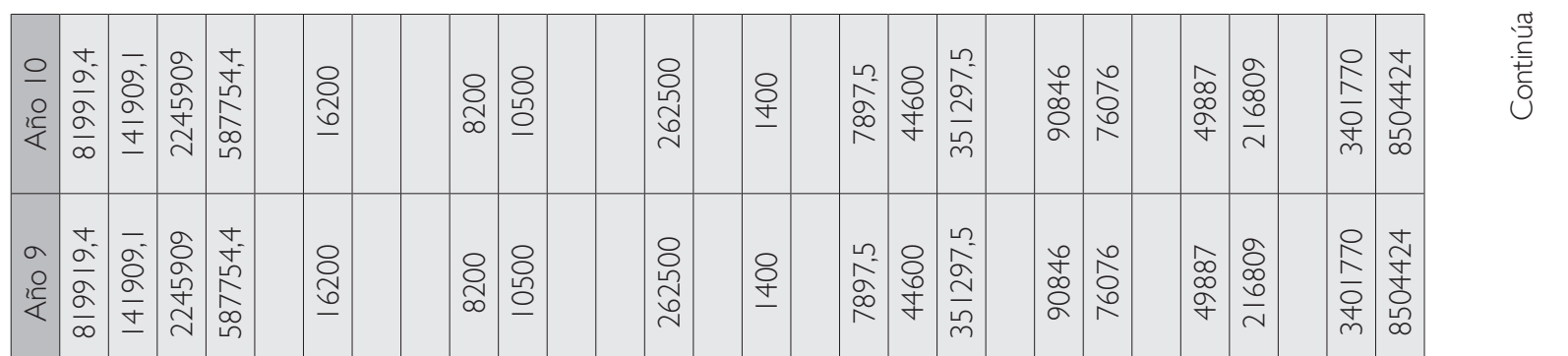

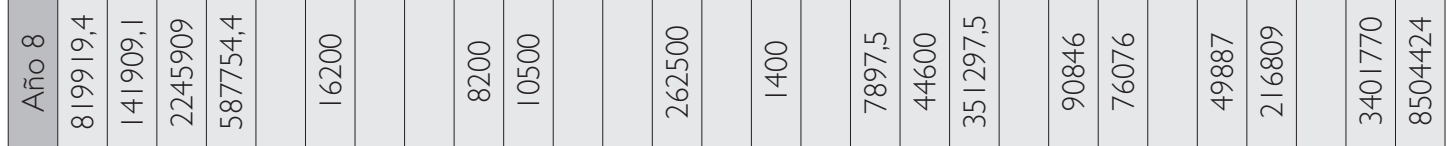

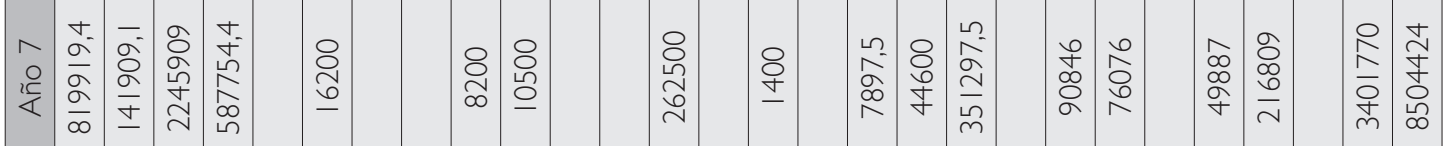

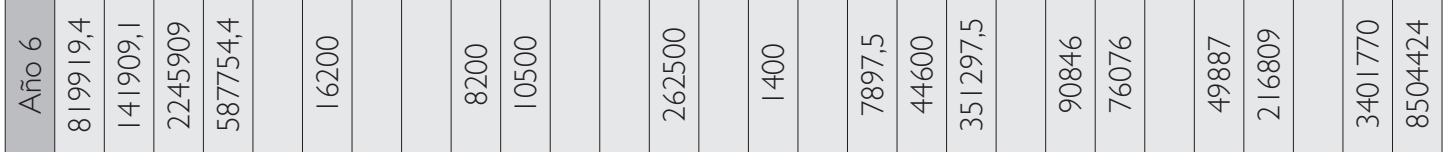

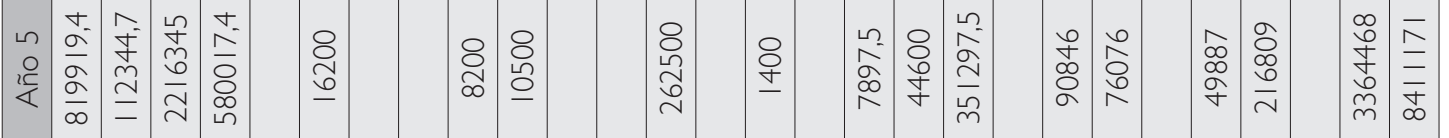

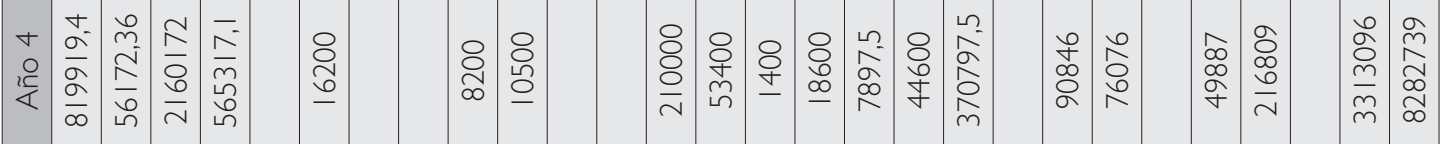

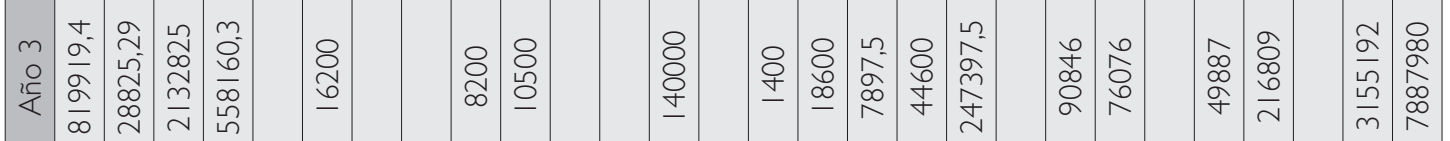

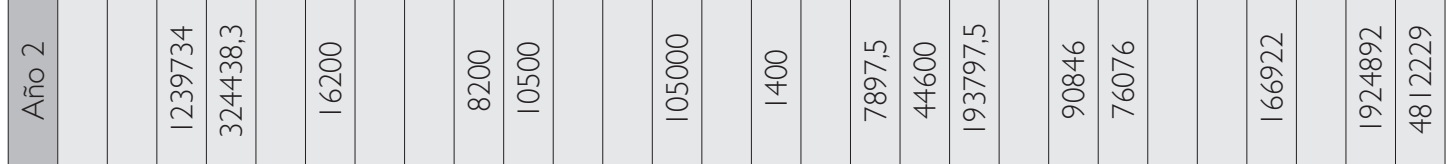

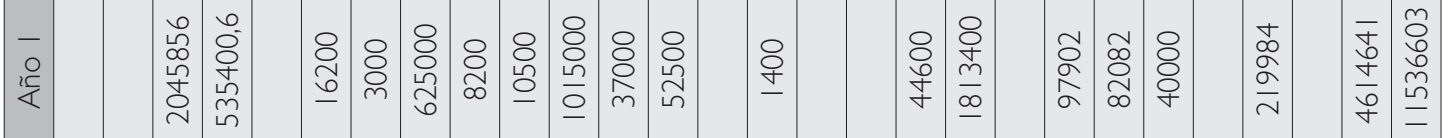

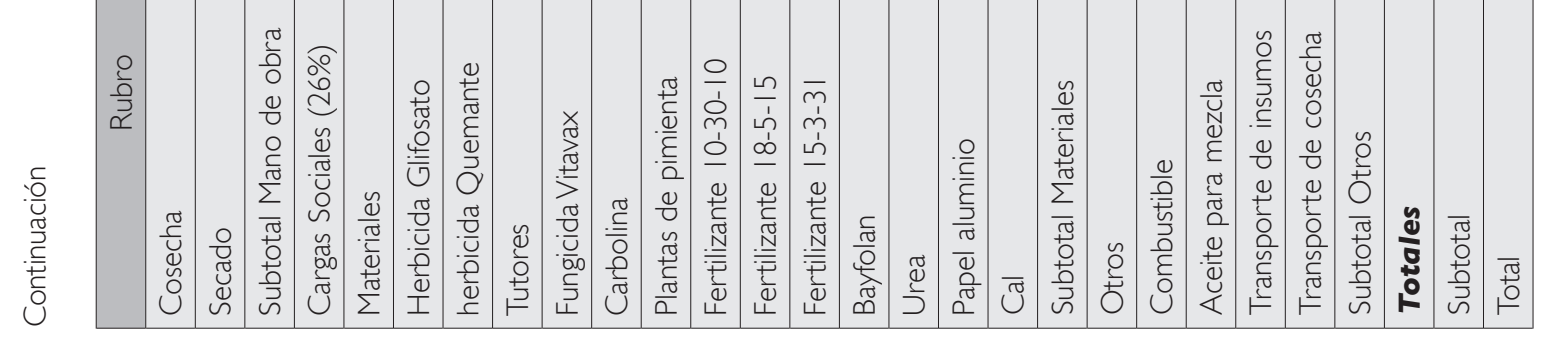


Cuadro 3. Costos máximos (colones) de producción aceptables para el proyecto de pimienta. Costa Rica. Elaboración propia, 2012.

\begin{tabular}{|c|c|c|}
\hline Año & Costo base $(\phi)$ & Costo máximo $(\not)$ \\
\hline 1 & 11536602 & 14047055 \\
\hline 2 & 479.729 & 7302181 \\
\hline 3 & 7867479 & 10377932 \\
\hline 4 & 8262239 & $1077269 \mid$ \\
\hline 5 & 8390670 & 10901123 \\
\hline 6 al 10 & 8483924 & 10994376 \\
\hline
\end{tabular}

Se analizó la sensibilidad que podría presentar el VAN en caso de que la producción, el precio y los costos aumenten o disminuyan un $20 \%$ o un $40 \%$.

EIVAN se considera sensible en el caso de que, ante una producción constante, los costos aumenten en un $40 \%$. Cuando los costos permanecen constantes pero el precio es el que disminuye, se va a presentar una situación de un VAN negativo, y esto se daría en caso de que el precio llegue a bajar 20\% con respecto al precio pagado al productor.

Del análisis anterior se evidencia que la variable que puede causar mayor variabilidad y efecto negativo en los indicadores financieros del proyecto es el precio, porque aunque los costos aumenten o la producción disminuya, si éste se mantiene constante, el proyecto mantendría la rentabilidad. En el cuadro 4 se presentan los flujos netos anuales del proyecto de proyección de pimienta para un período de 10 años, sin financiamiento.

\section{Conclusiones}

El productor dedicado al cultivo de pimienta cuenta con poco apoyo de las instituciones gubernamentales para recibir asistencia técnica.

El impacto ambiental que causa la actividad del cultivo de pimienta en El Roble de La Virgen es mínimo, ya que es amigable con el ambiente.

Las instituciones públicas o privadas relacionadas con el sector agropecuario no cuentan con registros de las diferentes variables de la producción de pimienta a nivel nacional.

El Roble de la Virgen, en Sarapiquí, presenta una adecuada localización para un proyecto de pimienta, ya que reúne las especificaciones necesarias para el desarrollo del cultivo.

Existe una demanda insatisfecha por parte de PROPICA, principal empresa compradora de la pimienta, por lo que hay posibilidad de ventas futuras.

PROPICA brinda estabilidad en cuanto a la negociación, debido al contrato y la fijación de precio mínimos.

El mercado nacional de la pimienta es muy inestable, debido a las variaciones en la producción y los precios internacionales.

El proyecto se considera factible según el análisis de sus principales indicadores financieros.

\section{Recomendaciones}

Por las características que tiene la pimienta cultivada en Costa Rica y por reunir los principales requerimientos para su cultivo, en la zona de El Roble de Sarapiquí es necesario que instituciones como el Ministerio de Agricultura y Ganadería (MAG) y el Consejo Nacional de Producción (CNP) desarrollen programas de fortalecimiento del sector, con el fin de incrementar las áreas y con ello los volúmenes de producción para que las familias obtengan mayores ingresos.

PROPICA podría establecer incentivos para estimular al productor que reúna las condiciones necesarias y así aumentar los volúmenes de producción.

Tal como se menciona en el estudio ambiental, es importante implementar el sistema de gaveteo en el terreno para aprovechar de mejor manera los residuos que son arrastrados con la lluvia y realizar una mejor conservación de suelos.

Se debe hacer un manejo planificado de la producción, que permita el aprovechamiento máximo de los recursos con los que cuenta el productor.

Es importante aumentar la variabilidad genética de los cultivos, de manera que se eviten problemas por ataques de plagas y enfermedades; así como también utilizar los insumos según los requerimientos de las plantas y determinados por estudios periódicos de suelo.

Con base en los indicadores financieros obtenidos en el estudio, se recomienda la implementación del proyecto de inversión en pimienta. 


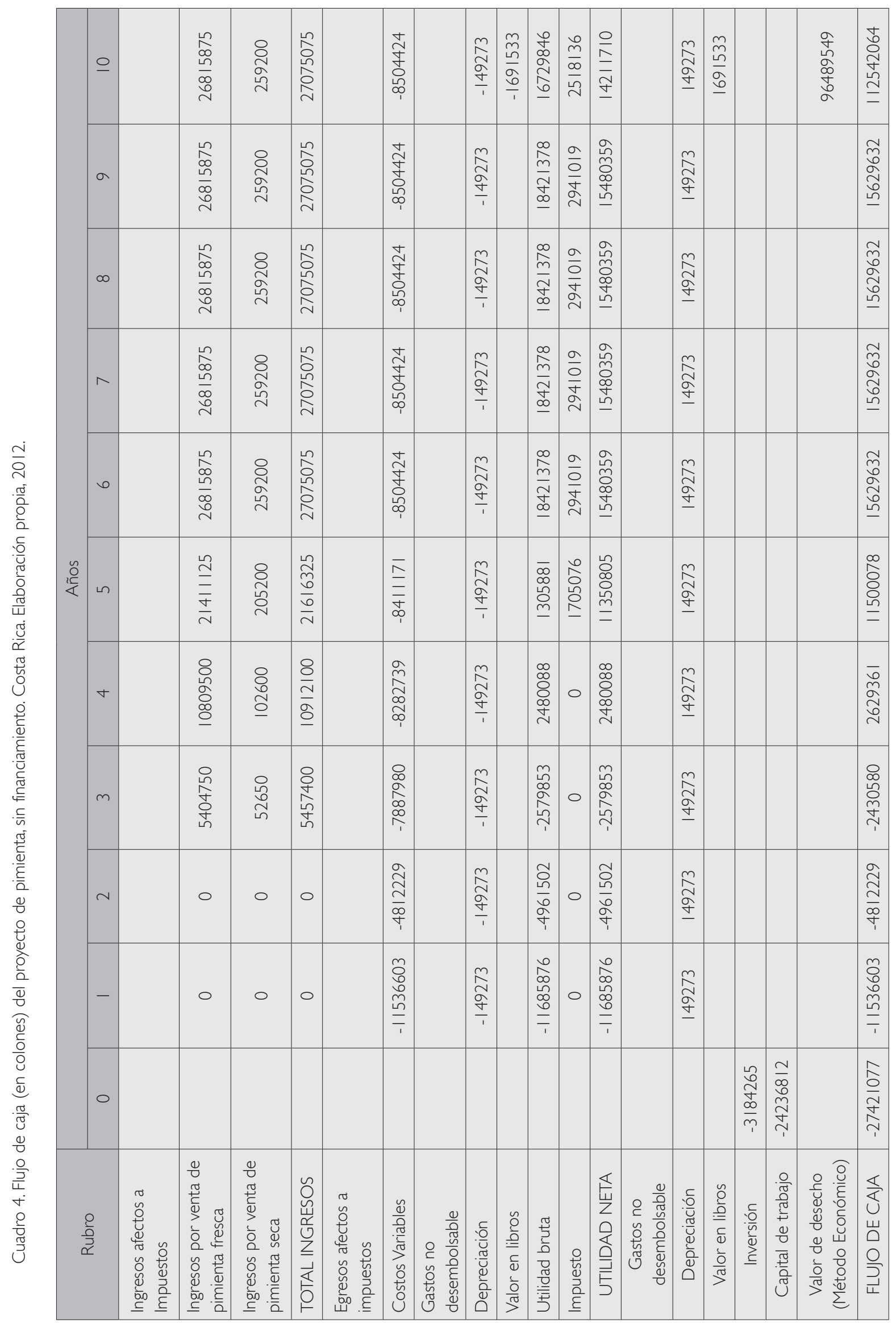




\section{Bibliografía}

Aguilar, E. \& Bogantes, A. (199I). Pimienta. In: MAG. Aspectos técnicos sobre cuarenta y cinco cultivos agrícolas de Costa Rica. San José, MAG, Dirección General de Investigación y Extensión Agrícola, pp. 59-66.

Bertsch, F. \& Méndez, J. (20 I I). Nutrición y fertilización de pimienta. San José: URC, CIA, ACCS.

CIASA (Consultorías Integradas Agropecuarias, S.A). (2007). Diagnóstico de la Agrocadena del cultivo de la Pimienta. Consultado el 12 junio 2012. Disponible en http://www. proyectopromes.org/userfiles/RES_I_I,I_2, I_4.PDF

EARTH (Escuela de Agricultura de la Región del Trópico Húmedo). (201 I). Proyecto Promes (AECID-EARTH) 201 I. Módulos Programa Acción-Producción para el fortalecimiento de productores(as) de pimienta: Proyecto Promes. Consultado el 14 junio 2012. Disponible en http://www.proyectopromes.org/userfiles/file/Programa\%20Accion-Produccion / modulo_I.pdf

FAO (Organización de las Naciones Unidas para la Alimentación y la Agricultura). (20I2). FAOSTAT. Consultado el 13 junio 2012. Disponible en http://faostat.fao.org/site/29//default. aspx

Sapag, N. \& Sapag, R. (2008). Preparación y evaluación de proyectos. 5 ed. Bogotá: McGraw-Hill Interamericana.

Segura, M. (2007). Caracterización de la cadena productiva de pimienta negra (Piper nigrum L.) en el cantón de Sarapiquí, Costa Rica. Consultado el 12 junio 2012. Disponible en http://usi.earth.ac.cr/glas/sp/dpg/55-2007.pd 